\title{
DNMT1 and Genomic Instability in Cancer
}

\section{Viviana Barra*}

Dipartimento Scienze e Tecnologie Biologiche Chimiche e Farmaceutiche (STEBICEF), University of Palermo, Palermo, Italy

*Corresponding author: Viviana Barra, Dipartimento Scienze e Tecnologie Biologiche Chimiche e Farmaceutiche (STEBICEF), University of Palermo, Palermo, Italy, Tel: 904-244-9361; Fax: 904-244-9361; E-mail: barra.viviana@gmail.com

Received date: 10 March 2014, Accepted date: 25 April 2014, Published date: 28 June 2014

Copyright: (c) 2014 Barra V, et al. This is an open-access article distributed under the terms of the Creative Commons Attribution License, which permits unrestricted use, distribution, and reproduction in any medium, provided the original author(s) and source are credited.

\begin{abstract}
DNA (cytosine 5-) MethylTransferase 1 (DNMT1) maintains the pre-existing methyl marks onto daughter strands by faithfully copying the methylation pattern from hemimethylated DNA during replication phase. The fundamental role of DNA methylation in cell physiology makes a controlled regulation of DNMT1 necessary. DNMT1 loss and global DNA hypomethylation are correlated with genomic instability in cancer. This mini review aims to provide a scenario of the mechanisms whereby DNMT1 dysfunction could induce genomic instability so as to clarify its functions and understand the dynamics of DNA methylation at cellular level with a focus on cancer.
\end{abstract}

Keywords: DNMT1; DNA methylation; Genomic instability

\section{Introduction}

DNA methylation is a fundamental epigenetic signal, cited as the "epigenetic writer" [1], that guarantees efficient temporal and spatial gene expression during cell life and development, and that insures the higher order of chromatin architecture. Imbalances of this process compromise genome integrity and its message throughout generations.

DNA methyltransferases (DNMTs) catalyse the transfer of a methyl-group from S-adenosyl-L-methionine (SAM) to the 5-position of cytosine residues placed almost exclusively at the so called " $\mathrm{CpG}$ islands", DNA regions that contain a high frequency of CpG sites. Four members of the DNMT family are known in humans (DNMT1, DNMT3a, DNMT3b and DNMT3L). All DNMTs have an N- and a Cterminal part separated by a KG repeat; the $\mathrm{N}$-terminus is the regulative part that contains a variable region and domains involved in DNA-binding, the C-terminus harbours usually the catalytic domain except for DNMT3L that, in fact, has not an associated enzymatic activity. DNMT3a and DNMT3b are "de novo" methyltransferases that establish DNA methylation pattern; only DNMT1 is responsible for the DNA methylation marks maintenance across successive cell divisions [2,3]. During DNA replication DNA is hemimethylated: the mother strand is methylated and the newly synthesised one is not. The protein UHRF1 (ubiquitin-like, containing PHD and RING finger domains 1) recognizes and binds the hemimethylated sites, then recruits DNMT1 that thereby methylates the newly synthesized DNA strand. The existence of a complex containing DNMT1, UHRF1 and PCNA (Proliferating Cell Nuclear Antigen), an auxiliary protein of DNA polymerase, shows the occurrence of DNA methylation during replication [4]. DNMT1 shows a 30 - to 40 -fold preference for hemimethylated sites [5], but the distinction between de novo and maintenance methylation is not so clear because DNMT1 de novo methylates non-CpG cytosines and to an extent even CpG islands [6] Moreover, human cancer cells showed cooperation between DNMT1 and DNMT3b in maintaining DNA methylation suggesting a more complex situation [7].
The importance of correct DNA methylation arises from the observation that DNMTs are essential for mouse viability $[3,8]$ and is emphasized by the high number of human diseases that are correlated to this epigenetic alteration. The paradox regarding DNA methylation pattern that shows both gene specific hypermethylation and genome wide hypomethylation is a characteristic of cancer cells, suggesting a role in human tumorigenesis [9].

Genomic instability is a distinguishing feature of the majority of human cancers and is considered a driving force for tumorigenesis. Various forms of genomic instability exist.

Chromosomal instability (CIN) implies structural and/or numerical changes of chromosomes; microsatellite instability (MSI or MIN) consists in alteration of the number of oligonucleotide repeats present in microsatellite sequences [10].

Global hypomethylation at repetitive sequences in cancer and DNMT1 reduction have been correlated to chromosomal instability, translocations, gene disruption and microsatellite instability [11]. In this short review we examine and outline the links between DNMT1 and genomic instability, and discuss the possible mechanisms of how they are orchestrated with a focus on cancer.

\section{DNMT1 and Chromosomal Instability}

Downregulation of DNMT1 is frequently observed in human tumors and is associated with DNA hypomethylation and genomic instability (i. e. chromosomal instability) [11-13]. Reduction of DNMT1 even of only $10 \%[14,15]$, or disruption of DNMT1/PCNA/ UHRF1 interactions, essential for DNMT1 recruitment onto DNA causes chromosome structural and numerical alterations [4]. The exact mechanism involving DNMT1 in CIN is not well understood yet, but the predominant hypothesis is that the DNMT1 reduction induces, through global DNA hypomethylation, loss of methylated marks at the repetitive sequences such as chromosome's pericentromeric region. The so created disorder would impair cellular processes such as DNA repair mechanisms and sister chromatids segregation triggering chromosomal instability. The essentiality of the interaction with PCNA/UHRF1 at the replication fork suggests that DNMT1 controls (and is highly controlled) in some way DNA replication. We 
demonstrated indeed that depletion of DNMT1 elicits a G1 arrest in normal fibroblasts. Only by overriding this arrest cells undergo chromosomal instability [14]. Accordingly, DNMT1 is regulated by important proteins that are part of cell cycle checkpoints. It has been showed that $\mathrm{pRb}$ promotes ATM/TIP60-dependent DNMT1 degradation and consequently pRb loss, accompanied by alterations in DNA damageresponse, results in DNMT1 deregulation and DNA methylation aberrations [16]. DNA hypomethylation would be induced by at least two alterations that on one side interfere with DNMT1 and on the other side allow the abrogation of the cellular response to DNA methyltransferase dysfunctions

\section{DNMT1 and Microsatellite Instability (MIN)}

DNMT1 loss in mouse ES has been correlated with increased microsatellite instability $[17,18]$. MIN phenotype is the result of impaired DNA Mismatch Repair (MMR) that corrects errors that spontaneously occur during DNA replication. Lots of evidences demonstrated the involvement of DNMT1 in MMR [17-19] through PCNA, probably due to the necessity of methylating repaired DNA [20]. Recently $\mathrm{Ha}$ et al showed that DNMT1 is implicated also in double strand breaks (DSB) repair system by interacting with RAD9 (member of 9-1-1 complex) and being recruited at damage site [21]. Taken together these results suggest a role for DNMT1 in the direct control of DNA health.

\section{Prospect}

DNA needs to be methylated in two moments, during its replication and after repair. In both DNMT1 is a key player not only for its DNA methyltransferase activity, but also for the control of these crucial events. Thus, DNMT1 activity during DNA replication and DNA repair is essential in order to control that everything is going well in the cell, reflecting the importance of DNA methylation for cell fitness. Conversely, DNMT1 dysfunctions will result in genome instability (CIN and MIN). Genomic instability is a characteristic of almost all human tumors and it has been suggested that it could not be a simple cancer capability but a feature that triggers all of the others (unlimited replication, deregulated growth signals, metastasis.) [10]. If DNMT1 controls genome stability in different contexts, it could be an important target of cancer cells. Loss of DNMT1 functional integrity or of its regulation could cause genomic instability acquisition/ propagation towards malignant transformation. In such a way understanding the fine mechanism(s) by which DNMT1 controls genome stability and is controlled itself would be interesting in order to develop a therapeutic strategy against genome instability on the road to cancer. Future perspective should be to understand if DNMT1 role in replication and repair is a structural or an active one, and/or the cell mechanisms that recognize DNMT1 alteration.

\section{Acknowledgement}

I am very grateful to prof. Aldo Di Leonardo (University of Palermo) for his valuable comments on the manuscript.

\section{References}

1. Kar S, Deb M, Sengupta D, Shilpi A, Parbin S, et al. (2012) An insight into the various regulatory mechanisms modulating human DNA methyltransferase 1 stability and function. Epigenetics 7: 994-1007.
2. Okano M, Xie S, Li E (1998) Cloning and characterization of a family of novel mammalian DNA (cytosine-5) methyltransferases. Nat Genet 19: 219-220.

3. Okano M, Bell DW, Haber DA, Li E (1999) DNA methyltransferases Dnmt3a and Dnmt3b are essential for de novo methylation and mammalian development. Cell 99: 247-257.

4. Hervouet E, Lalier L, Debien E, Cheray M, Geairon A, et al. (2010) Disruption of Dnmt1/PCNA/UHRF1 interactions promotes tumorigenesis from human and mice glial cells. PLoS One 5: e11333.

5. Jeltsch A (2006) On the enzymatic properties of Dnmt1: specificity, processivity, mechanism of linear diffusion and allosteric regulation of the enzyme. Epigenetics 1: 63-66.

6. Cheng X, Blumenthal RM (2008) Mammalian DNA methyltransferases: a structural perspective. Structure 16: 341-350.

7. Rhee I, Bachman KE, Park BH, Jair KW, Yen RW, et al. (2002) DNMT1 and DNMT3b cooperate to silence genes in human cancer cells. Nature 416: 552-556.

8. Li E, Bestor TH, Jaenisch R (1992) Targeted mutation of the DNA methyltransferase gene results in embryonic lethality. Cell 69: 915-926.

9. Robertson KD (2005) DNA methylation and human disease. Nat Rev Genet 6: 597-610.

10. Negrini S, Gorgoulis VG, Halazonetis TD (2010) Genomic instability--an evolving hallmark of cancer. Nat Rev Mol Cell Biol 11: 220-228.

11. Watanabe Y, Maekawa M (2010) Methylation of DNA in cancer. Adv Clin Chem 52: 145-167.

12. Eden A, Gaudet F, Waghmare A, and Jaenisch R (2003). Chromosomal instability and tumors promoted by DNA hypomethylation. Science (New York) N.Y 300; 455.

13. Karpf AR, Matsui S (2005) Genetic disruption of cytosine DNA methyltransferase enzymes induces chromosomal instability in human cancer cells. Cancer Res 65: 8635-8639.

14. Barra V, Schillaci T, Lentini L, Costa G, Di Leonardo A (2012) Bypass of cell cycle arrest induced by transient DNMT1 post-transcriptional silencing triggers aneuploidy in human cells. Cell Div 7: 2 .

15. Gaudet F, Hodgson JG, Eden A, Jackson-Grusby L, Dausman J, et al. (2003) Induction of tumors in mice by genomic hypomethylation. Science 300: 489-492.

16. Shamma A, Suzuki M, Hayashi N, Kobayashi M, Sasaki N, et al. (2013) ATM mediates pRB function to control DNMT1 protein stability and DNA methylation. Mol Cell Biol 33: 3113-3124.

17. Kim M, Trinh BN, Long TI, Oghamian S, Laird PW (2004) Dnmt1 deficiency leads to enhanced microsatellite instability in mouse embryonic stem cells. Nucleic Acids Res 32: 5742-5749.

18. Wang KY, James Shen CK (2004) DNA methyltransferase Dnmt1 and mismatch repair. Oncogene 23: 7898-7902.

19. Loughery J E, Dunne P D, O'Neill K M, Meehan R R, McDaid J R, et al. (2011). DNMT1 deficiency triggers mismatch repair defects in human cells through depletion of repair protein levels in a process involving the DNA damage response. Human molecular genetics 20, 3241-3255.

20. Mortusewicz O, Schermelleh L, Walter J, Cardoso MC, Leonhardt $\mathrm{H}$ (2005) Recruitment of DNA methyltransferase I to DNA repair sites. Proc Natl Acad Sci U S A 102: 8905-8909.

21. Ha K, Lee GE, Palii SS, Brown KD, Takeda Y, et al. (2011) Rapid and transient recruitment of DNMT1 to DNA double-strand breaks is mediated by its interaction with multiple components of the DNA damage response machinery. Hum Mol Genet 20: 126-140. 